\title{
Preferred Strategies for Female and Male Initiators In Romantic Relationship Initiation: The Role of Stereotypes Related to Romantic Relationships, Rejection Sensitivity and Relationship Anxiety
}

\author{
Mediha Ömür \\ medihaomur@gmail.com
}

Ayda Büyükşahin-Sunal

Ankara University

\author{
Doi:10.5901/jesr.2015.v5n1s1p195
}

\section{Abstract}

\begin{abstract}
The aim of this study is to evaluate the associations between preferences about which strategy (e.g. direct, indirect or pasive strategies) should be used by women and men in order to initiate relationship, attitudes toward stereotypes related to romantic relationships, rejection sensitivity and relationship anxiety. It is also aimed to evaluate to what extent rejection sensitivity and relationship anxiety determine the stereotypical attitudes concerning men's initiation in relationships. The sample of the study included 373 participants (228 women - 145 men) between the ages of 18 - $37(X=22.08, S D=2.18)$. The questionaire that consisted of demografic information form, Relationship Fear/Anxiety Subscale of Multidimensional Relationship Scale, Attitudes Toward Stereotypes Related to Romantic Relationships Scale and Rejection Sensitivity Scale were administered to the participants in person as printed forms or by internet as online forms. Findings showed that both female and male participants mostly supported indirect strategy for female initiators, and direct strategy for male ones. Female participants' support for direct strategy was found to be greater than male participants' support for direct strategy where the initiator was male $(p<.05)$. Conversely males' support for direct strategy was greater than female participants' support for direct strategy where the initiator was female $(p<.05)$. Relationship anxiety, attitudes toward stereotypes related to romantic relationships and rejection sensitivity were found to be differed in the basis of different strategy preferences for female and male initiators. Female participants who supported indirect strategy for female initiators were more in favor of stereotypes concerning men's initiation and men's dominance in relationships than female participants who supported direct strategy for female initiators $(p<$ .01). These participants were also found to have more rejection sensitivity than the other female participants group by a marginal significance level $(p=.051)$. Male participants who supported direct strategy for female initiators were found to have more relationship anxiety than male participants who supported indirect strategy for female initiators $(p<.01)$. Male participants who supported direct strategy for male initiators were observed as more supportive of stereotypes concerning men's dominance in relationships $(p<.01)$. In women sample, supporting stereotypes concerning men's initiation was explained by the number of individual's own initiations and rejection sensitivity. Findings of the research were discussed within the framework of gender roles, stereotypes related to romantic relationships and rejection sensitivity theory.
\end{abstract}

Keywords: Romantic Relationship, Relationship Initiation, Rejection Sensitivity, Stere

The beginning of a relation in which couples get to know each other is a duration that is of paramount importance in the development of the relationship (Berscheid \& Garizano, 1979; Snyder, Berscheid \& Glick, 1985). As Clark, Shaver and Abrahams (1999) suggested, even though many studies concerning maintanence of relationships are carried out, the number of studies, in which the initiation of these relationships are of concern, are very limited This study aims to investigate the associations between the views about the strategies that men and women are supposed to follow and the stereotypes involving romantic relationships, rejection sensitivity and relationship anxiety In addition, an investigation into the extend to which rejection sensitity and relationship anxiety predicts the stereotypical attitudes associated with men being supposed to be active and initiative is aimed.

The expression relationship initiation refers to the beginning stage of close relationships (Sprecher \& Guynn, 2009). According to Clark and colleagues (1999), during the realtionship initiation period, individiuals employ different strategies to interact or deepen their interaction with people they like or are attracted to. These are:

(a) becoming emotionally involved (revealing personal information), (b) directly initiating a relationship (making physical contact, directly asking a potential partner to start a relationship), (c) signaling indirectly (hinting, talking 
generally about romance), (d) manipulating the situation (making the setting romantic, maintaining close physical contact), (e) joking (teasing, playfully insulting), (f) demonstrating resources (gift-giving, showing off possessions), (g) using third parties to initiate a relationship (getting friends or family members to assist), and ( $h$ ) acting passively (waiting for the other person to make the first move) (p. 712).

These strategies might be both direct or indirect and the person might even choose to remain completely inactive and wait. (Clark et. al. 1999, Vorauer \& Ratner 1996)

When the studies involving relationship initiation periods are analysed, it is possible to see that the main focus of attention has been on gender. In studies by Clark and colleagues (1999) it has been observed that while men are more active, employing direct strategies, women tend to be more passive, employing indirect strategies. Findings suggesting that men are more active initiative than women while woman employ more indirect ways have been observed in some other studies as well (i.e., Berger, 1987; Kelley \& Rolker-Dolinsky, 1987; MacGregor \& Cavallo, 2011). The expectation that men are supposed to initiate the relationship and exhibit dominance while women employ coyness and inhibition to sex, can be commonly observed in Turkey as well as many western cultures (Eaton \& Rose, 2011; Sakallı \& Curun, 2001; Sakallı-Uğurlu, 2003; Schleicher \& Gilbert, 2005). Even when couples define themselves as equalitarians they tend to follow more traditional methods (Ganong \& Coleman, 1992). These findings point to the extend to which these stereotypes involving relationship initiation and other periods are effective. In addition, Sakallı and Curun (2001) claim that stereotypes concerning relationships involve two dimesions, male dominance and male assertiveness, and that women are more in favour of male assertiveness when it comes to relationship initiation. In studies conducted in Western cultures, the view that men should be assertive has been found to be more favoured among women (Schleicher \& Gilbert, 2005).

Sensitivities regarding rejection may be of influence on why the stereotypes about the man being the initiator has been embraced more by women. Both men and women may prefer the opposite side to be more assertive in order to avoid the threat and anxiety of rejection. Therefore, the stereotypes that suggest man should be initiators may find more support among women. Even though it is known that sensitivities about rejection may inhibit indivuals from being initiators (Vorauer \& Ratner, 1996), no research investigating how these sensitivities support the stereotype of the man being assertive has been done.

Some claim that sensitivities about rejection form based on the relationship with primary caregivers (Downey, Bonica \& Rincon, 1999; Feldman \& Downey, 1994). According to the rejection sensitivity theory (Downey et. al.,1999; Feldman \& Downey, 1994) that includes this view, the experiences of rejection and neglect in interactions with the primary caregiver may cause the individual to be over sensitive to clues of rejection as well as being in a nervous expectation concerning rejection in their later relationships. On the other hand London, Downey, Bonica \& Paltin (2007) suggested that rejection sensitivity is related to social anxiety and withdrawal. This function of rejection sensitivity may cause the individual to prefer the opposite sex to be assertive in initiation of a romantic relationship. Therefore, women's being highly in favour of the stereotypes involving men's initiation assertiveness may be due to their rejection sensitivites.

In addition to rejection sensitivity, anxieties and fears towards developing an intimate relationship may also affect relationship initiation assertiveness. These anxieties concerning relationship initiation have been studied in terms of attachment styles in attachment theory studies (Hazan \& Shaver, 1987; Bartholomew \& Horowitz, 1991). According to this theory, secure attachment style is when a positive and consistent relationship has formed during infancy with the primary caregiver which in turn reflects in adulthood as forming and maintaining positive relationships. Insecure attachment styles such as anxious-avoidant attachment (Hazan \& Shaver, 1987) and fearful attachment (Bartholomew \& Horowitz, 1991) suggest negative and inconsistent relationships with the primary caregiver in infancy and maintaining these fears and anxities about relationships in adulthood. Individuals with secure attachment style may be expected to be more assertive in intimate relationships (Hazan \& Shaver, 1987). In that case, relationship anxiety which is a reflection of insecure attachment style, might be expected to negatively affect assertiveness in initiating the romantic relationship. However, findings are required to validate this expectation.

Towards the elimination of these shortcomings mentioned earlier in relationship initiation studies, this study aims to investigate the romantic relationship initiation strategies that are deemed appropriate for male and female initiators in terms of stereotypes, rejection sensitivity and relationship anxiety. In addition, an investigation into whether some demographic variables, attitudes towards stereotypes suggesting men are to be dominant, rejection sensititvity and relationship anxiety predict the stereotypes that suggest men are to be assertive, is aimed. This way, the reasons why women are more in favour of the stereotype that men are supposed to be more assertive in relationship initiation, has been investigated. 


\section{Method}

\subsection{Participants}

The sample included 373 university students from different public and private universities in Turkey between the ages of $18-37$ of which $61,1 \%$ were women $(n=228)$, and $38,9 \%$ were men $(n=145)$. The mean age was 22,08 .

\subsection{Materials}

Personal Information Form: This form was consisted of questions about age, university, bereducational levels of the parents, the number of previous romantic relationships and the number of romantic relationships which participant himself/herself initiated. Additionally, this form included questions concerning preferred strategies for male and female initiators (direct, indirect or passive strategies).

The Relationship Fear/Anxiety Subscale of The Multi-Dimensional Relationship Questionaire: This questionaire was developed to assess several psychological experiences related to intimate relationships by Snell, Schicke and Arbeiter (2002). Turkish adaptation of this questionaire was conducted by Buyuksahin (2005). The Relationship Fear/Anxiety Dimension is a subscale of this questionaire and includes ten items which can be scored from 1 to 5 .

Attitudes Toward Stereotypes Related To Romantic Relationships Scale: First form of this scale which had 12 items was developed by Sakallı and Curun (2002). The scale was shortened to 10 items by Sakallı in 2003. The short form assesses the supportive attitudes toward stereotypes relevant to male assertiveness and male dominance in romantic relationships. In this study, both dimensions (male assertiveness and male dominance) were used. Items of the scale can be scored from 1 to 7 .

Rejection Sensitivity Questionaire: This questionaire was developed to measure individuals' levels of rejection sensitivity by Downey and Feldman (1996). Erözkan (2004) adapted this questionaire to Turkish culture. The questionaire has 18 items that can be scored from 1 to 8 . Total score of the questionaire provides the rejection sensitivity level.

\subsection{Procedure}

Firstly, Ethical Committee Approval was obtained from Ankara University and then the questionaires were distributed via internet or by hand. Printed questionaire forms were administered in Ankara University Language-History and Geography Campus and in a elective psychology course at Aydin University. The participants who took the printed questionaire forms were presented with an Informed Consent Form and requested to sign it after reading. The link of the website which lead participants to online form was announced through a social medium (facebook). In order to prevent repeated participations the users which had the same IP numbers were removed from the data. It took 10-15 minutes to fill in the whole research questionaire.

\section{Results}

The main aim of this study was to evaluate the preferred strategies for female and male romantic relationship initiators. For this purpose, firstly, most preferred initiation strategies for females and males were investigated. Secondly, it was examined whether these preferences differentiated in terms of gender. Finally, the variables which predict the supportive attitudes toward stereotypes relevant to male dominance in romantic relatonships were explored through a stepwise regression analysis because of the main concern of the study.

\section{Findings of Demographic Variables}

Gender comparisions of parental educational levels were performed through a Mann-Whitney $U$ test because the independent variable is ordinal. Findings showed no gender difference concerning educational level of the participants mothers $(U=15397, p<.05, r=-.98)$. Similarly, male and female partcipants did not differ in educational levels of the fathers $(U=16052, p<.05, r=-.08)$.

The female $(X=3,43)$ and the male participants $(X=3,92)$ were found to not have significantly different number of previous romantic relationships $(t[366]=-1,1, p<.05)$. However, the number of the relationships which was initiated by 
participant himself/herself was found to be greater in males $(X=2,73)$ than females $(X=0,66)(t[159,58]=-6,95, p<$ .001). Additionally, female participants $(X=23.56)$ were found to be more supportive of male initiation than male participants $(X=21.79)$ (t $[369]=3.17, p<.01)$; while male participants $(X=25.06)$ were more supportive of male dominance in romantic relationships than female participants $(X=17.29)(t[365]=-10.66, p<.01)$. No significant difference was found in terms of rejection sensitivity between female $(X=54.84)$ and male participants $(X=52.72)(t$ $[361]=1.24, p>05)$. However, marginally significant gender difference was observed in relationsip anxiety variable $(t$ $[362]=1.83, p=.07)$. That is, female participants $(X=23.03)$ scored higher than male ones $(X=21.54)$.

\subsection{Finding of Preferred Strategies For Female and Male Initiators}

It was shown that indirect strategy (e.g. "to stimulate desired potential partner to make the first move") was mostly preferred for female initiators both by female and male participants. Eighty-one percent of females and $62,1 \%$ of males agreed that women should stimulate men to make the first move. Least preferred initiation strategy was found to be passive strategy (e.g. " just to wait doing nothing") for women initiators ( $5,3 \%$ in females and $5,5 \%$ in males).

For male initiators, direct strategy (to make the first move asking out) was the most preferred strategy by both female and male participants. Ninety-four percent of the female participants and $81,4 \%$ of the male participants supported the idea of making the first move to initiate a relationship with the desired potential partner. Passive strategy was the least preferred way of initiation for male initiators among both female $(0,4 \%)$ and male participants $(4,1 \%)$. It was also found that the preference of direct strategy for female initiators was more common in male participants $(z$ [371] $=$ $4,09, p<.05)$; while the preference of direct strategy for male initiators was more common in female participants ( $z$ [371] $=3,47, p<.05)$.

Data of the participants who preferred the passive strategy for male or female initiators was not included in the comparisons of rejection sensitivity, attitudes toward sterotypes related to romantic relationships and relationship anxiety in terms of preferred strategies for male and female initiators because of the insufficent sample size to perform the necessary analyses. So, the data which belonged to 12 females and 8 males was removed in the comparision of these variables in terms of pereferences for male initiators; and the data which belong to 1 female and 6 males was removed in the comparision of these variables in terms of pereferences for female initiators. The data of the rest of the participants who preferred indirect or direct strategies was used to evaluate associations between different strategy preferences and rejection sensitivity, attitudes toward sterotypes related to romantic relationships and relationship anxiety variables.

The female participants who preferred the indirect strategy for female initiators were found to be more supportive of both stereotypes of male assertiveness $(U=2026.50, p<.01, r=-2.75)$ and male dominance $(U=1147, p<.01, r=$ 5.50) in romantic relationships than the other female participants who preferred the direct strategy for female initiators. These participants were also found to be more sensitive to rejection in a marginal level of significance $(U=2256, p=$ $.051, r=-1.95)$ than the ones who preferred direct strategy for female initiators. No significant difference was found in relationship anxiety between the females who preferred the direct and indirect strategies $(U=2552, p>.05, r=-1.07)$.

Male participants who preferred the indirect strategy for female initiators scored lower in relationship anxiety than those who preferred the direct strategy for female initiators $(U=1594.50, p<.05, r=-2.10)$. Male participants showed no significant difference in rejection sensitivity $(U=1995.50, p>.05, r=-.13)$, attitudes toward male assertiveness $(U=$ 1947.50, $p>.05, r=-.36)$ and male dominance $(U=1818.50, p>.05, r=-1.25)$ in romantic relationships in terms of preferences of the direct or indirect strategies for female initiators.

No significant difference was found in the women sample in relationship anxiety $(U=1204, p>.05, r=-.66)$, rejection sensitivity $(U=1172, p>.05, r=-.81)$, attitudes toward male assertiveness $(U=1039.50, p>.05, r=-1.51)$ and dominance $(U=1189.50, p>.05, r=-.80)$ in romantic relationships in terms of preferences of the direct or indirect strategies for male initiators. The male participants who preferred the direct or indirect strategies for male initiators did not show any significant difference in relationship anxiety $(U=1017.50, p>.05, r=-1.15)$, rejection sensitivity $(U=$ 1098.50, $p>0.05, r=-.66)$ and attitudes toward male assertiveness $(U=1000.50, p>.05, r=-1.36)$ in romantic relationships. However, male participants who prefferred the indirect strategy for male initiators were found to be less supportive of attitudes toward male dominance $(U=1594.50, p<.01, r=-2.198)$ than those who preferred the direct strategy.

\subsection{Findings of Regression Analyses}

For exploration of the predictors of attitudes toward stereotype concerning male assertiveness in romantic relationships, 
stepwise hierarchical regression analyses were performed both for all participants together and males and females seperately. On these analyses, age, educational level of the mother and father, the number of previous romantic relationships and the number of previous romantic relationships which was initiated by the participant himself/herself were entered in the regression first. Relationship anxiety and rejection sensitivity variables were entered in the second block.

The results of the regression analysis which were performed for all of the participants regardless of heir gender are presented in Table 1. As can be seen in Table 1, the number of previous romantic relationships which was initiated by the participant were found to be a significant predictor of the dependent variable $\left(F[1,343]=13.22 ; R^{2}=.04, \beta=-.19, t\right.$ $[343]=-3.64 ; p<.01)$. In the second block, rejection sensitivity significantly predicted the dependent variable $(F[1,342]=$ 9.93; $\mathrm{R}^{2}=.06, \beta=.14, \mathrm{t}[342]=2.54 ; \mathrm{p}<.01$ ). The number of previous romantic relationships which was initiated by the participant explained $4 \%$ of the variance and the addition of rejection sensitivity variable raised explained variance to $6 \%$.

The results of the regression analysis which was performed for only the female participants are presented in Table 2. As seen in Table 2, the number of previous romantic relationships which were initiated by the participant were found to be a significant predictor of the dependent variable $\left(F[1,208]=9.41 ; R^{2}=.04, \beta=-.21\right.$, t $\left.[208]=-3.07 ; p<.01\right)$. In the second block, rejection sensitivity significantly predicted the dependent variable $\left(F[1,207]=22.62 ; R^{2}=.07, \beta=.16, t\right.$ $[207]=2.38 ; p<.01)$. The number of previous romantic relationships which were initiated by the participant explained $4 \%$ of the variance and with the addition of rejection sensitivity variable explained variance rose to $7 \%$. In the regression analysis which was performed only for the male participants, none of the first and second block variables had a significant predictive role in attitudes toward men's assertiveness in relationships.

\section{Discussion}

Findings involving the demographic variables investigated in the study show that the number of relationships in which men are initiators are more than those that women initiate. This shows that the stereotypes (Eaton \& Rose, 2011; Sakallı \& Curun, 2001) which suggest men are to be initiators in romantic relationships, affect actual dating behaviours. Moreover, the finding that the male assertiveness stereotype which involves the view that men are supposed to be initiators finds more favour among women is consistent with prior studies (Sakallı \& Curun, 2001; Sakallı-Uğurlu 2003).

In literature concerning intimate relationships, findings which suggest females have higher rejection sensitivity have been observed while there were no differences between genders involving relationship anxiety (Büyükşahin, 2005; Erözkan 2004). In this study, however, different results about rejection sensitivity and relationship anxiety have been found. Rejection sensitivity not changing among genders while relationship anxiety is observed to be more among women in a marginally significant manner point to a possibility that the relation between these variables and gender may change over time.

In the study, both men and women have supported the view that when women like someone, they should enable the opposite sex to make the first move. The view that men should make the first move in initiation period was the most preferred strategy for male initiators among both men and women. In other words, there is a consensus that women should act indirectly and men have to act directly. This shows that, as stated in Eaton and Rose (2011), traditional gender roles which put the active role in initiation to men is still widely accepted in our time. Furthermore, while the view that women should be more direct in initiation has found favour more among men, the view that men should be more direct in initiation has found more favour among women. This can be interpreted as both men and women want the opposite sex to be more assertive in relationship initiation. As mentioned earlier, the stereotype involving male assertiveness to be more favourable among women also points out to this.

Analyses concerning the roles of relationship anxiety, attitudes towards stereotypes related to romantic relationships and rejection sensitivity in preferred initiation strategies for men and women have resulted differently among men and women.

For women who prefer indirect strategies for other women, supporting male assertiveness and dominance in romantic relationships is in relation with the negative views of stereotypical attitudes involving relationships towards a woman making the first move. In addition to stereotypical attitudes, rejection sensitivity has been observed to be lower with women who support a more direct strategy for other women. This finding suggests that women's not supporting other women to make the first move is influenced by the fear of rejection. Fear of rejection may stop the individual from disclosing to the person they like or from taking the first step (Vorauer \& Ratner, 1996). The view that men should take the first step not women could be due to women's fear of rejection. As McDaniel (2005) mentioned earlier, women more advocate traditinal role of men in initiating relationships because this allows them tol essen the risk of rejection. 
Accordingly, the results of the stepwise regression analysis predicts that the rejection sensitivity in women supports the male assertiveness stereotype in relationships.

For men who had different preferences involving female initiation strategies, no significant difference has been noted on their attitudes towards stereotypes involving relationships or on rejection sensitivity. On the other hand, men who think women should employ a more direct strategy for initiation are found to have higher relationship anxitey compared to the men who believe women should employ a more indirect strategy. These findings show that for men , seeing it appropriate for a woman to take the first step is related to the fears and anxieties related to having a relationship. This is not suprising, however, when it is considered that individuals with secure attachment are more assertive in intimate relationships compared to those who have insecure attachement and that relationship anxiety is a reflection of insecure attachment. Supporting assertiveness in the opposite sex could be considered an indicator of the persons own lacking in assertiveness and this lacking could be a reflection of the attachment style.

The initiation strategies that men prefer for other males is found to be in relation with only supporting the stereotypes which suggest men are to be dominant. Accordingly, more support for the stereotype which suggests man are to be dominant is observed among males who believe other males should employ direct strategies, compared to those who believe other males should employ indirect strategies. Attitudes towards stereotypes that suggest man should be assertive, rejection sensitivty and relationship anxiety were found to have no relation to the strategies preferred by men for other males.

Although the stereotype that suggets men are supposed to be assertive in a relationship includes the view that men are supposed to take the first step, interestingly, the values obtained from this variable is not higher in men who prefer a direct strategy for other This shows that the attitudes towards male assertiveness in a relationship and the attitudes towards whether a man takes the first step when he likes someone or not are not the same. The first represents a must for a romantic relationship while the latter is an expression of attitudes on what must be done when someone is liked. The individual might believe it to be a good idea to take the first step when someone is liked, although, he may at the same time not support the idea that its a man's duty to initiate. In literature, a man wanting a woman to be more assertive has been interpreted as a wish to reduce their burden in the relationship (Scharlott \& Christ, 1995; Sakallı \& Curun, 2001). The findings of this study show that, this wish of men doesn't necessarily mean that they will avoid taking the first step when they like someone.

As it is known, the well established gender roles in society encourage men in romantic relationships to be sexually active while making it known, comfortably approach the person they like and show dominant behaviour in the relationship (Eaton \& Rose, 2011; Schleicher \& Gilbert, 2005). In romantic relationships, supporting male dominance and prefering direct strategies for other males can be related to each other in terms of the courage that the gender roles provide men with. Keeping this in mind, this courage does not stop men from wanting the opposite sex to be more assertive and share the burdens of the relationship (Scharlott \& Christ, 1995; Sakallı \& Curun; 2001).

\section{References}

Bartholomew, K. \& Horowitz, L. M. (1991). Attachment styles among young adults : A test of a four- category model. Journal of Personality and Social Psychology, 61, 226-244.

Berger, C. R. (1987). Planning and scheming: Strategies for initiating relationships. In R. Burnett, P. McGhee \& D.Clarke (Ed.), Accounting for relationships: Social representations of interpersonal links (pp. 158-174). London: Methuen.

Berscheid, E. \& Graziano, W. G. (1979). The initiation of social relationships and interpersonal attraction. In R.L. Burgess ve TL Huston (Ed.), Social Exchange in Developing Relationships. London: Academic Pres.

Büyükşahin, A. (2005). Çok boyutlu ilişki ölçeği: Geçerlik ve güvenirlik çalışması. Türk Psikiyatri Dergisi, 16(2), 97-105.

Clark, C. L., Shaver, P. R. \& Abrahams, M. F. (1999). Strategic behaviors in romantic relationship initiation. Personality and Social Psychology Bulletin, 25, 709-722.

Downey, G., Bonica, C. \& Rincon, C. (1999). Rejection sensitivity and adolescent romantic relationships. In W. Furman, B. Brown ve C. Feiring (eds.), The development of romantic relationships in adolescence (pp. 148-174) New York: Cambridge Press.

Downey, G. \& Feldman, S. (1996). Implications of rejection sensitivity for intimate relationships, Journal of Personality and Social Psychology, 70(5), 1327-1343.

Eaton, A. A. \& Rose, S. (2011). Has heterosexual dating become more egalitarian? A 35-year review using Sex Roles. Sex Roles, 64 (11-12), 843-862.

Erözkan, A. (2004). Romantik ilişkilerde reddedilmeye dayalı incinebilirlik bilişsel değerlendirme ve başaçıkma, Unpublished Doktorate Thesis, KTÜ-SBE, Trabzon.

Feldman, S. \& Downey, G. (1994). Rejection sensitivity as a mediator of the impact of the childhood exposure to family violence on adulthood attachment behavior. Developmental Psychopathology, 6, 231-247. 
Ganong, L. \& Coleman, M. (1992). Gender differences in self and future partner expectations. Journal of Family Issues, $13,55-64$.

Hazan, C. \& Shaver P. (1987). Romantic love conceptualized as an attachment process. Journal of Personality and Social Psychology, 52, 511-524.

Kelley, K. \& Rolker-Dolinsky, B. (1987). The psychosexology of female initiation and dominance. In D. Perlman ve S. Duck (Ed.), Intimate relationships: Development, dynamics, and deterioration (pp. 63-87). Newbury Park, CA: Sage.

London, B., Downey, G., Bonica, C. \& Paltin, I. (2007). Social causes and consequences of rejection sensitivity. Journal of Research on Adolescence, 17(3), 481-506.

MacGregor, J. C. D. \& Cavallo, J. V. (2011). Breaking the rules: Personal control increases women's direct relationship initiation. Journal of Social and Personal Relationships, 28(6), 848-867.

McDaniel, A. K. (2005). Young women's dating behavior: Why/why not date a nice guy? Sex Roles, 53, 347-359.

Sakallı, N. \& Curun, F. (2001). Romantik ilişkilerle ilgili kalıpyargılara karşı tutumlar. Tecrübi Psikoloji Çalışmaları, 22, 31-45

Sakalli-Uğurlu, N. (2003). How do romantic relationship satisfaction, gender stereotypes, and gender relate to future time orientation in romantic relationships? Journal of Psychology, 137(3), 294-303.

Scharlott, B.W. \& Christ, W. G. (1995). Overcoming relationship-initiation barriers: The impact of a computer-dating system on sex role, shyness, and appearance inhibitions. Computers in Human Behavior, 11(2), 191-204.

Schleicher, S. S. \& Gilbert, A. L. (2005). Heterosexual dating discourses among college students: Is there still a double standard? Journal of College Student Psychotherapy, 19(3), 12-13.

Snell, W. E. Jr, Schicke, M. \& Arbeiter, T. (2002). The multidimensional relationship questionnaire: psychological dispositions associated with intimate relations. İçinde: WE Snell Jr. (Ed.), New Directions in The Psychology of Intimate Relations: Research and Theory. Cape Girardeau, MO: Snell Publications.

Snyder, M., Berscheid, E. \& Glick, P. (1985). Focusing on the exterior and the interior: Two investigations of the initiation of personal relationships. Journal of Personality and Social Psychology, 48, 1427-1439.

Sprecher, S. (1985). Sex differences in bases of power in dating relationships. Sex Roles, 12, ss. 449-462.

Vorauer, J. D. \& Ratner, R. K. (1996). Who's going to make the first move? Pluralistic ignorance as an impediment to relationship formation. Journal of Social and Personal Relationships, 13(4), 483-506.

\section{Tables}

Table 1. Results of The Stepwise Hierarchical Regression Analysis For The Predictors of Attitudes Toward Stereotypes Related To Male Assertiveness in Romantic Relationships (All Participants)

\begin{tabular}{|c|c|c|c|c|}
\hline Variables (According to enterance order) & $\mathrm{R}^{2}$ s.d. $\quad \mathrm{F}$ & B & StandartError & $\beta$ \\
\hline Step I (Demografic Variables) Personally initiated relationships & $.041-34313.22$ & -.40 & .11 & $-.19-3.64$ \\
\hline Step II Rejection sensitivity & $061-3429.93$ & .05 & .02 & .142 .56 \\
\hline
\end{tabular}

Table 2. Results of The Stepwise Hierarchical Regression Analysis For The Predictors of Attitudes Toward Stereotypes Related To Male Assertiveness in Romantic Relationships (Female Participants)

\begin{tabular}{|c|c|c|c|c|c|c|c|}
\hline Variables (According to enterance order) & R2 & s.d. & $\mathrm{F}$ & $B$ & Standart Error & $\beta$ & $\mathrm{t}$ \\
\hline Step I (Demografic Variables) Personally initiated relationships & .04 & 1-208 & 9.41 & -.97 & .32 & -.21 & -3.07 \\
\hline Step II Rejection sensitivity & .07 & $1-207$ & 7.65 & .06 & .02 & .16 & 2.38 \\
\hline
\end{tabular}

